\title{
Hubbard Model with Nearest-Neighbor and Bond-Charge Interaction: Exact Ground-State Solution in a Wide Range of Parameters
}

\author{
Rainer Strack and Dieter Vollhardt \\ Institut für Theoretische Physik C, Technische Hochschule Aachen, D-5100 Aachen, Federal Republic of Germany
}

(Received 26 January 1993)

\begin{abstract}
We present the exact ground-state wave function and energy of a general Hubbard model with kinetic energy $t$ and on-site, nearest-neighbor, and bond-charge interaction $U, V, X$, respectively, for a wide, physically relevant range of parameters with $X=t$ on lattices with arbitrary coordination numbers $Z$ at half filling.
\end{abstract}

PACS numbers: $75.10 . \mathrm{Jm}, 71.10 .+\mathrm{x}, 71.28 .+\mathrm{d}$

The Hubbard model $[1,2]$ is the generic model for interacting electrons in narrow-band systems. Indeed, it is the simplest model that is able to describe both the band and the atomic limit. As shown by Hubbard in 1963 this model may be obtained from the general Hamiltonian

$\hat{H}=\sum_{\mathbf{i j}, \sigma} T_{\mathbf{i j}} \hat{c}_{\mathbf{i} \sigma}^{\dagger} \hat{c}_{\mathbf{j} \sigma}+\frac{1}{2} \sum_{\mathbf{i j k} l, \sigma \sigma^{\prime}}\left(\mathbf{i j}\left|\frac{1}{r}\right| \mathbf{k} \boldsymbol{l}\right) \hat{c}_{\mathbf{i} \sigma}^{\dagger} \hat{c}_{\mathbf{j} \sigma^{\prime}}^{\dagger} \hat{c}_{l \sigma^{\prime}} \hat{c}_{\mathbf{k} \sigma}$

by approximating the interaction term. Here, $\hat{c}_{\mathbf{i} \sigma}^{\dagger}\left(\hat{c}_{\mathrm{i} \sigma}\right)$ creates (annihilates) a particle with spin $\sigma$ in a Wannier orbital localized at site $\mathbf{i}$; the corresponding number operator is $\hat{n}_{\mathbf{i} \sigma}=\hat{c}_{\mathrm{i} \sigma}^{\dagger} \hat{c}_{\mathbf{i} \sigma}$. The parameter $T_{\mathbf{i j}}$ is the Fourier transform of the band energy $\epsilon_{\mathbf{k}}$, and $(\mathbf{i j}|l / r| \mathbf{k} l)$ is the matrix element of the Coulomb interaction with respect to the Wannier functions at sites $\mathbf{i}, \mathbf{j}, \mathbf{k}, \boldsymbol{l}$. For narrowband systems the dominant matrix elements of the interaction are

$$
\begin{aligned}
& U \equiv(\mathbf{i i}|1 / r| \mathbf{i i}), \\
& V \equiv(\mathbf{i j}|1 / r| \mathbf{i j}), \quad(\mathbf{i}, \mathbf{j}) \text { nearest neighbors } \\
& X \equiv(\mathbf{i i}|1 / r| \mathbf{i j}), \quad(\mathbf{i}, \mathbf{j}) \text { nearest neighbors }
\end{aligned}
$$

where $U$ and $V$ parametrize the on-site and a nearestneighbor density interaction, respectively, and $X$ is a bond-charge interaction [3-8], which may be viewed as a density-dependent nearest-neighbor hopping [9]. For $3 d$ electrons in transition metals Hubbard estimated these matrix elements as $U \approx 20 \mathrm{eV}, V \approx 6 \mathrm{eV}, X \approx 0.5 \mathrm{eV}$, where $V$ will be reduced to $2-3 \mathrm{eV}$ by metallic screening [1]. This estimate motivated him to take into account only the on-site interaction and neglect all other contributions. One is thus led to the famous Hubbard model $[1,2]$. In spite of its utmost simplicity it is thought to describe much of the essential physics of narrow-band systems. The Hubbard model has attracted immense interest during the last decades, and an exact solution in closed form was obtained for $d=1$ [10]. However, it is still not known to what extent the inclusion of the neglected interaction terms will change the physics described by the Hubbard model. In particular, $V$ and $X$ cannot always be expected to be much smaller than the on-site interaction $U$. For example, for benzene the parameters were estimated as $U \approx 17 \mathrm{eV}, V \approx 9 \mathrm{eV}, X$ $\approx 3.3 \mathrm{eV}[4-6]$. In any case, even for $V, X \ll U$ the very presence of interactions which directly couple nearestneighbor sites should lead to new effects.

When the nearest-neighbor $(V)$ and the bond-charge interaction $(X)$ are taken into account one obtains the following generalized Hubbard model:

$$
\begin{aligned}
\hat{H} & =\hat{T}+\hat{U}+\hat{V}+\hat{X} \\
& =-t \sum_{\langle\mathbf{i}\rangle\rangle, \sigma}\left(\hat{c}_{\mathbf{i} \sigma}^{\dagger} \hat{c}_{\mathbf{j} \sigma}+\hat{c}_{\mathbf{j} \sigma}^{\dagger} \hat{c}_{\mathbf{i} \sigma}\right)+U \sum_{\mathbf{i}} \hat{n}_{\mathbf{i} \uparrow} \hat{n}_{\mathbf{i} \downarrow}+V \sum_{\langle\mathbf{i}\rangle, \sigma \sigma^{\prime}} \hat{n}_{\mathbf{i} \sigma} \hat{n}_{\mathbf{j} \sigma^{\prime}}+X \sum_{\langle\mathbf{i j}\rangle, \sigma}\left(\hat{c}_{\mathbf{i} \sigma}^{\dagger} \hat{c}_{\mathbf{j} \sigma}+\hat{c}_{\mathbf{j} \sigma}^{\dagger} \hat{c}_{\mathbf{i} \sigma}\right)\left(\hat{n}_{\mathbf{i}-\sigma}+\hat{n}_{\mathbf{j}-\sigma}\right),
\end{aligned}
$$

with $t, U, V, X \geq 0$. The kinetic energy $\hat{T}$ is due to hopping between nearest neighbors with matrix element $t$. The index $\langle\mathbf{i j}\rangle$ stands for summation over the nearestneighbor pairs. For $X=0$ the Hamiltonian reduces to the so-called "extended Hubbard model." The generalized model (3) has been considered previously by several groups [3-8]; an exact solution for general parameters is not known. Since $t$ is typically of the order of $0.2-2 \mathrm{eV}$ the values of $X$ and $t$ are seen to be quite comparable; most recently this was explicitly shown for a square lattice of oxygen ions representing a $\mathrm{CuO}_{2}$ plane [8].

In this Letter we show that for $X=t$ the ground state of the model (3) can be obtained exactly in two parameter regimes. In particular, we will present the exact ground-state wave function and energy of the model on a three-dimensional subspace of the four-dimensional parameter space $(t, U, V, X)$ in the half-filled band case $n=1$. We investigate two different regimes, corresponding to two different inequalities for the parameters, where in the first case the ground-state is obtained for an arbitrary lattice and in the second case for an arbitrary bipartite lattice. We consider lattices with a constant number of nearest neighbors $Z$.

The exact solution is made possible by the fact that for $X=t$ hopping processes interfere in such a way that the number of doubly occupied sites is conserved, i.e., $\left.[\hat{T}+\hat{X}, \hat{U}]\right|_{t=x}=0$, but $\left.[\hat{T}+\hat{X}, \hat{V}]\right|_{t=x} \neq 0$. Models with 
a similar kind of effective hopping have recently been investigated in some detail [11,12]. However, we will later show that exact results may even be obtained when the commutator does not vanish.

For $X=t$ the Hamiltonian (3) may be written in two equivalent forms:

$$
\begin{aligned}
\hat{H} & =-2 t Z L(1-\hat{n})+(U-4 t Z-V Z) \sum_{\mathbf{i}} \hat{n}_{\mathbf{i} \uparrow} \hat{n}_{\mathbf{i} \downarrow}+t \hat{R}_{+}+t \hat{S}_{+}+\frac{V}{2} \hat{Y} \\
& =-2 t Z L \hat{n}+\frac{1}{Z}(V Z-U-4 t Z) \sum_{\langle\mathbf{i j}\rangle, \sigma \sigma^{\prime}} \hat{n}_{\mathbf{i} \sigma} \hat{n}_{\mathbf{j} \sigma^{\prime}}+t \hat{R}-+t \hat{S}_{-}+\frac{U+4 t Z}{2 Z} \hat{Y},
\end{aligned}
$$

where $L$ is the number of lattice sites and $\hat{n}=(1 / L)$ $\times \sum_{\mathbf{i}, \sigma} \hat{n}_{\mathbf{i} \sigma}$. The operators $\hat{R}_{ \pm}, \hat{S}_{ \pm}$, and $\hat{Y}$ are given by

$$
\begin{aligned}
& \hat{R}_{ \pm}=\sum_{\langle\mathrm{ij}\rangle, \sigma} \hat{P}_{\mathrm{ij} \sigma, \pm}^{\dagger} \hat{P}_{\mathrm{ij} \sigma, \pm} \\
& \hat{S}_{ \pm}=\sum_{\langle\mathbf{i j}\rangle, \sigma} \hat{Q}_{\mathrm{ij} \sigma, \pm \hat{Q}_{\mathrm{ij} \sigma, \pm}^{\dagger}} \\
& \hat{Y}=\sum_{\langle\mathbf{i j}\rangle} \hat{N}_{\mathrm{ij}}\left(\hat{N}_{\mathrm{ij}}-1\right),
\end{aligned}
$$

where $\hat{P}_{\mathbf{i j} \sigma,+}=\hat{C}_{\mathbf{i} \sigma}^{\dagger}+\hat{C}_{\mathrm{j} \sigma}^{\dagger}, \hat{P}_{\mathrm{ij} \sigma,-}=\hat{C}_{\mathbf{i} \sigma}-\hat{C}_{\mathbf{j} \sigma}, \hat{Q}_{\mathbf{i j} \sigma,+}=\hat{\zeta}_{\mathbf{i} \sigma}$ $+\hat{\zeta}_{\mathbf{j} \sigma}$, and $\hat{Q}_{\mathrm{ij} \sigma,-}=\hat{\zeta}_{\mathbf{i} \sigma}^{\dagger}-\hat{\zeta}_{\mathbf{j} \sigma}^{+}$with $\hat{C}_{\mathbf{i} \sigma}=\hat{c}_{\mathbf{i} \sigma}\left(1-\hat{\eta}_{\mathbf{i}-\sigma}\right)$, $\hat{\zeta}_{\mathbf{i} \sigma}=\hat{c}_{\mathbf{i} \sigma} \hat{n}_{\mathbf{i}-\sigma}$, and $\hat{N}_{\mathbf{i j}}=\sum_{\sigma}\left(\hat{n}_{\mathbf{i} \sigma}+\hat{n}_{\mathbf{j} \sigma}\right)$.

In the following we will consider two different parameter regimes. We will derive upper and lower bounds on the exact ground-state energy and show that they coincide. This well-known method for finding exact ground states has also been applied recently to correlated electrons in the special limit $U=\infty[13,14]$.

I. regime: $U-(4 t+V) Z \geq 0$. Since $\hat{R}_{+}, \hat{S}_{+}$are positive semidefinite operators and $\langle\hat{Y}\rangle \geq Z L\left(2 n^{2}-n\right)$, which follows from the Schwarz inequality, a lower bound on the energy is given by $E_{l}=-2 t Z L(1-n)+V Z L\left(n^{2}\right.$ $-n / 2$ ) for a density $n$. To obtain an upper bound we consider the wave function

$$
\left|\Psi_{1}\right\rangle=\prod_{\mathbf{i} \in \mathcal{A}} \hat{c}_{\mathbf{i} \dagger}^{\dagger} \prod_{\mathbf{j} \in \mathcal{A}^{\prime}} \hat{c}_{\mathbf{j} \downarrow}^{\dagger}|0\rangle
$$

where $|0\rangle$ is the vacuum and $\mathcal{A}, \mathcal{A}^{\prime}$ are arbitrary disjoint sets of lattice sites which together build up the total lattice. This wave function corresponds to a density $n=1$ and consists of singly occupied sites. One can easily verify that $\left|\Psi_{I}\right\rangle$ is an eigenfunction of $\hat{H}$ with eigenvalue $E_{u}=V Z L / 2$, which is thus an upper bound on the exact ground-state energy. Since upper and lower bound coincide for $n=1$, the exact ground-state energy of the generalized Hubbard model (3) for $X=t, U-(4 t+V) Z$ $\geq 0$, and $n=1$ on an arbitrary lattice with coordination number $Z$ is simply given by

$$
E=V Z L / 2 \text {. }
$$

The corresponding ground state, which is $2^{L}$-fold degenerate, describes a paramagnetic insulator. The set of ground-state wave functions (6) is complete.

We should like to stress that the restriction imposed by the inequality on the parameters is not unphysical. For example, the exact solution applies in the case of a cubic lattice $(Z=6)$ with parameter values $t=X=0.5 \mathrm{eV}$, $V=2 \mathrm{eV}, U \geq 24 \mathrm{eV}$ which is very close to the values estimated above [1]. For a small deviation $\delta t=t-X \neq 0$ and a bipartite lattice an antiferromagnetic configuration, i.e., spin-density wave (SDW), is immediately favored [15]; this is what one would expect from the pure Hubbard model for large $U$ and $n=1$.

II. regime: $U+(4 t-V) Z \leq 0$. Since $\hat{R}$ - and $\hat{S}$ - are positive semidefinite operators, too, a lower bound on the energy is given by $E_{l}=-2 t Z L n+(U+4 t Z) L\left(n^{2}-n / 2\right)$. The wave function

$$
\left|\Psi_{I I}\right\rangle=\prod_{i \in \mathcal{A}} \hat{c}_{i \dagger}^{\dagger} \hat{c}_{i \downarrow}^{\dagger}|0\rangle
$$

defined on an arbitrary bipartite lattice with $\mathcal{A}$ as one of the two sublattices, is an eigenfunction of $\hat{H}$ with eigenvalue $E_{u}=U L / 2$; clearly $E_{u}$ is an upper bound on the exact ground-state energy. Since upper and lower bound coincide for $n=1$, the exact ground-state energy of the generalized Hubbard model (3) for $X=t, U+(4 t-V) Z$ $\leq 0$, and $n=1$ on an arbitrary bipartite lattice with coordination number $Z$ is given by

$$
E=U L / 2 \text {. }
$$

The corresponding ground state (8), which is unique apart from a twofold degeneracy due to the two sublattices, describes a nonmagnetic insulator. It corresponds to a charge-density wave (CDW) with maximal order parameter ("chessboard phase," see Fig. 1). For a simple cubic lattice the above exact solution is valid, for example, for $t=X=0.5 \mathrm{eV}, V=4 \mathrm{eV}, U \leq 12 \mathrm{eV}$.

In Fig. 2 the $(U / t, V / t, X / t)$ ground-state phase diagram for a hypercubic lattice is shown. For $X / t=1$ we solved the model exactly in the two shaded regions. For $V Z \geq U+4 t Z$ a charge-density wave (CDW) and for

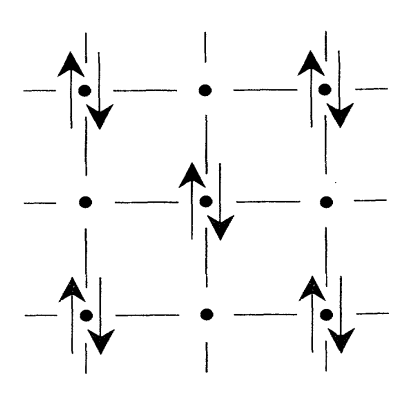

FIG. 1. The exact ground-state configuration of the general Hubbard model (3) for $X=t, U+(4 t-V) Z \leq 0$, and $n=1$ on a square lattice. 


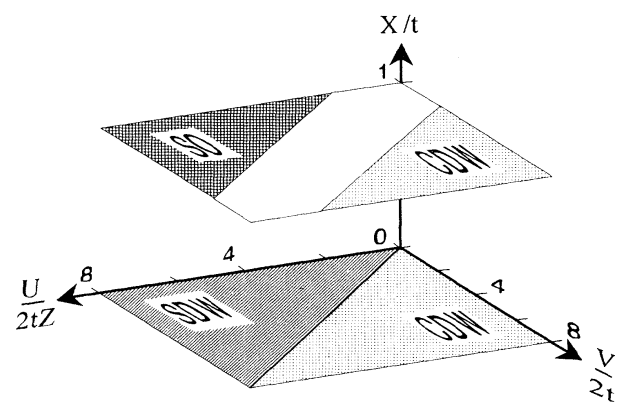

FIG. 2. $(U / t, V / t, X / t)$ ground-state phase diagram for the general Hubbard model (3) on a hypercubic lattice. The exact result for $X / t=1$ is shown in comparison with approximate results for $X / t=0$ (see text).

$U \geq V Z+4 t Z$ a configuration with singly occupied (SO) sites is found. This should be compared with results for $X / t=0$ (extended Hubbard model) obtained by perturbation theory in high dimensions [16] where one finds a transition between a SDW and a CDW state at $U=V Z$. Monte Carlo results for a one-dimensional system show a qualitatively similar behavior with, however, a small deviation in the transition line $U=V Z$ [17]. Obviously the latter results are qualitatively very similar to the above exact results for $X / t=1$ (note that the SO state will immediately switch to a SDW state for $X / t \neq 1$ ).

In the conventional Hubbard model $(X=0, V=0)\left|\Psi_{I}\right\rangle$ is a ground-state wave function only for $U=\infty$. In the generalized Hubbard model with $X=t$ this ground state is already realized at finite $U$ with $U \geq 4 t Z+V Z$. Similarly, while in the extended Hubbard model $(X=0)\left|\Psi_{I I}\right\rangle$ is the ground-state wave function only for $V=\infty$, in the generalized Hubbard model with $X=t$ this ground state is already realized at finite $V$ with $V Z \geq 4 t Z+U$. Hence, $\hat{X}$ stabilizes the two regimes down to a finite value of $U$ and $V$, respectively.

Our method can be also applied to the insulating regime of the Hamiltonian recently investigated by Montorsi and Rasetti [11]. This model is a (rather unrealistic) generalization of (3) to spin-dependent hopping amplitudes $t^{\sigma, \sigma^{\prime}}=X^{\sigma, \sigma^{\prime}}=t$ and $V=0$. The model can be mapped onto a Falicov-Kimball-like Hamiltonian [18]

$$
\begin{aligned}
\hat{H}_{\mathrm{MR}}= & t \sum_{\langle\mathbf{i j}\rangle}\left(\hat{c}_{\mathbf{i} \uparrow}^{\dagger} \hat{c}_{\mathbf{j} \uparrow}+\hat{c}_{\mathbf{j} \uparrow}^{\dagger} \hat{c}_{\mathbf{i} \uparrow}\right)\left(1-\hat{n}_{\mathbf{i} \downarrow}-\hat{n}_{\mathbf{j} \downarrow}\right) \\
& +U \sum_{\mathbf{i}} \hat{n}_{\mathbf{i} \uparrow} \hat{n}_{\mathbf{i} \downarrow},
\end{aligned}
$$

where only the up-spin electrons are mobile $(t, U \geq 0)$. By investigating the limit $Z \rightarrow \infty, U=\infty$, and $n<1$ Janiš et al. [19] showed that the supposedly exact solution derived in [11], displaying a Mott transition, is not exact. Nevertheless, using exact diagonalization and Monte Carlo methods Michielsen, De Raedt, and Schneider [20] recently showed that in dimensions $d=1,2$ the model (10) does exhibit a metal-insulator transition for $n=1$ at
$U=4 d t$. The analytic approach outlined above allows us to check these results. For this we rearrange the Hamiltonian as

$$
\begin{aligned}
\hat{H}_{\mathrm{MR}}= & -t Z L(1-\hat{n})+(U-2 t Z) \sum_{\mathbf{i}} \hat{n}_{\mathbf{i} \uparrow} \hat{n}_{\mathbf{i} \downarrow} \\
& +t \hat{P}+t \hat{Q}
\end{aligned}
$$

with positive semidefinite operators $\hat{P}$ and $\hat{Q}$ which have a similar form as that of $\hat{R}_{+}, \hat{S}_{+}$. For $n=1$ and $U \geq 2 t Z$ and for arbitrary lattices the exact ground state is then found to be given by $\left|\Psi_{I}\right\rangle$, (6), with energy $E=0$. Hence, the system is indeed insulating for all $U \geq 2 t Z$ on an arbitrary lattice. The numerical results [20] for $Z=2,4$ are thus seen to agree with the exact result. Although our method is limited to the insulating phase one may speculate that, by analogy, in the general Hubbard model (3) with $X=t$ the system exhibits a metal-insulator transition at $U-(4 t+V) Z \approx 0$ and $U+(4 t-V) Z \approx 0$, too.

Our method can be also applied to the generalized Hubbard model (3) for negative $U, V$. In this case one finds phase separation for $X=t, U \leq-4 t Z$, and $V<0$ [21].

A further extension of (3) is obtained by including the interaction term (ii $|1 / r| j j$ ) which corresponds to hopping of doubly occupied sites $[1,4]$. For $X=t$ and in the regime II the Hamiltonian (4b) can be cast into a form with an effectively attractive on-site interaction; hence we expect a superconducting state for $n<1$.

The generalized Hubbard model (3) may also be investigated for arbitrary spin degeneracy $N_{\sigma}$. In this case the exact ground state can be obtained for $n=1, X=t$, and $U-2 t Z N_{\sigma}-V Z \geq 0$ (singly occupied sites), as well as for $n=N_{\sigma} / 2, X=t /\left(N_{\sigma}-1\right)$ and $U-V Z+2 t Z N_{\sigma} /\left(N_{\sigma}\right.$ $-1)^{2} \leq 0$ (charge-density wave) [21]. Note that the number of doubly occupied sites is no longer conserved for $N_{\sigma}>2$, i.e., the commutator of the effective-hopping Hamiltonian $\hat{T}+\hat{X}$ with $\hat{U}$ no longer vanishes in this case.

In summary, we obtained the exact ground-state solution for a general Hubbard model including nearestneighbor and bond-charge interaction for arbitrary coordination number $Z$ in a wide, physically relevant range of parameters. This ground state is either a highly degenerate state with singly occupied sites, or corresponds to a charge-density wave. These results may be used as a starting point for perturbation theory in $\delta t=t-X \neq 0$.

We wish to thank P. G. J. van Dongen, V. Janiš, G. S. Uhrig, and R. Vlaming for helpful discussions. One of us (R.S.) gratefully acknowledges a scholarship of the Studienstiftung des Deutschen Volkes. This work was supported in part by the Sonderforschungsbereich 341 of the Deutsche Forschungsgemeinschaft.

[1] J. Hubbard, Proc. R. Soc. London A 276, 238 (1963).

[2] M. C. Gutzwiller, Phys. Rev. Lett. 10, 159 (1963); J. Kanamori, Prog. Theor. Phys. 30, 275 (1963).

[3] J. E. Hirsch, Physica (Amsterdam) 158C, 326 (1989); J. 
E. Hirsch and F. Marsiglio, Phys. Rev. B 41, 2049 (1990); F. Marsiglio and J. E. Hirsch, Phys. Rev. B 41, 6435 (1990).

[4] S. Kivelson, W. P. Su, J. R. Schrieffer, and A. J. Heeger, Phys. Rev. Lett. 58, 1899 (1987); 60, 72 (1988).

[5] D. Baeriswyl, P. Horsch, and K. Maki, Phys. Rev. Lett. 60, 70 (1988).

[6] J. T. Gammel and D. K. Campbell, Phys. Rev. Lett. 60, 71 (1988); D. K. Campbell, J. T. Gammel, and E. Y. Loh, Phys. Rev. B 42, 475 (1990).

[7] A. Painelli and A. Girlando, Phys. Rev. B 39, 2830 (1989).

[8] J. Appel, M. Grodzicki, and F. Paulsen, Phys. Rev. B 47, 2812 (1993).

[9] Note that the off-diagonal interaction $X$ (denoted by $\Delta t$ in Refs. $[3,8])$ is referred to as a "bond-charge-site-charge" interaction in Ref. [6].

[10] E. H. Lieb and F. Y. Wu, Phys. Rev. Lett. 20, 1445 (1968).
[11] A. Montorsi and M. Rasetti, Phys. Rev. Lett. 66, 1383 (1991).

[12] F. H. L. Essler, V. E. Korepin, and K. Schoutens, Phys. Rev. Lett. 68, 2960 (1992); 70, 73 (1993).

[13] U. Brandt and A. Giesekus, Phys. Rev. Lett. 68, 2648 (1992).

[14] R. Strack, Phys. Rev. Lett. 70, 833 (1993).

[15] For $t, V, X \ll U$ and $n=1$ the model (3) reduces to an effective Heisenberg model with antiferromagnetic coupling constant $4(t-X)^{2} / U$; see also Refs. $[3,6]$.

[16] P. G. J. van Dongen, Phys. Rev. Lett. 67, 757 (1991).

[17] J. E. Hirsch, Phys. Rev. Lett. 53, 2327 (1984).

[18] L. M. Falicov and J. C. Kimball, Phys. Rev. Lett. 22, 997 (1969).

[19] V. Janiš, F. Gebhard, R. Strack, and D. Vollhardt, Phys. Rev. Lett. 69, 2443 (1992).

[20] K. Michielsen, H. De Raedt, and T. Schneider, Phys. Rev. Lett. 68, 1410 (1992).

[21] R. Strack (to be published). 